\title{
Influencia de la auto-eficacia social y el apoyo social en la calidad de las relaciones de pareja adolescentes
}

\author{
Francisca Wals, Eva M. Romera* y Carmen Viejo \\ Universidad de Córdoba, España
}

(Recibido 9 Febrero, 2015; Aceptado 25 Febrero, 2015)

RESUMEN: Este estudio analiza las características de las relaciones de pareja adolescentes y la influencia que tiene la auto-eficacia social y el apoyo social en su calidad. un total de 209 estudiantes ( $49.3 \%$ chicos) de entre $12-18$ años ( $X=14.28$; D.T $=1.21$ ), escolarizados en tres centros de Educación Secundaria Obligatoria de Córdoba, cumplimentaron un cuestionario de auto-informe formado por preguntas abiertas que informaban del sexo, edad, nivel educativo y situación sentimental, y por un conjunto de instrumentos medidos en escala Likert que exploraban la calidad de las parejas, la auto-eficacia y el apoyo social. Los análisis descriptivos y correlacionales señalaron alta implicación en las relaciones sentimentales, mostrando más satisfacción y expectativas de futuro los chicos y los participantes de mayor edad. Se detecta que los/as jóvenes más auto-eficaces contaban con más habilidades para manejar las exigencias de una relación romántica. Se discuten los resultados en base a la importancia que tienen las relaciones positivas dentro del grupo de iguales y el apoyo social respecto a la calidad de estas parejas.

Palabras clave: adolescencia, relaciones románticas, relaciones entre iguales, apoyo social, auto-eficacia social.

Influence of self-efficacy and social support in the quality of teen couple relationships

\begin{abstract}
This study analyses the characteristics of adolescent relationships and influence that has social self-efficacy and social support. A number of 209 youth $(49.3 \%$ boys $)$ aged between $12-18$ years $(X=14.28$; D.T. $=1.21)$, enrolled in three centers of compulsory secondary education of Cordoba, completed a selfreport consisting of open-ended questions that reported the sex, age, educational level and relationship status, and a set of instruments measured on Likert scale that explored the quality of couples, self-efficacy and social support. Descriptive and correlational analysis indicated a high involvement in romantic relationships, showing more satisfaction and future expectatives boys and older participants. It is detected that teenagers with more social self-efficacy had more skills to handle the demands of a relationship. We discuss the results based on the importance that have positive peer group relationships and social support with respect to the quality of these couples.
\end{abstract}

Keywords: adolescence, romantic relationships, peer relationships, social support, social self-efficacy. 


\section{INTRODUCCIÓN}

En la adolescencia se espera que se cumplan tareas de desarrollo específicas relacionadas con comportamientos, emociones y conocimientos que exigen constituir nuevas y más maduras relaciones sociales fuera del contexto familiar, y para lograr con éxito dichas tareas el adolescente adecua sus patrones de comportamiento para adaptarse a una realidad social caracterizada por la influencia de los iguales (Gorrese y Ruggieri, 2012). En estas edades comienzan además a formarse las primeras relaciones de pareja, donde el aprendizaje de nuevas formas de relacionarse afectivamente asume un rol significativo (Stenberg, 2008).

Las relaciones románticas adolescentes tradicionalmente han sido consideradas triviales, transitorias y carentes de compromiso (Collins, 2003). Sin embargo, numerosas investigaciones muestran como las relaciones de pareja adolescentes pueden satisfacer las necesidades sexuales, de afiliación, apego y cuidados. En la medida en que vaya transcurriendo la adolescencia la pareja irá ascendiendo en la jerarquía de apego, las relaciones serán más duraderas y estables, con mayor intimidad y transmitirán mayor seguridad y apoyo (Tani y Fonzi, 2005).

La forma en la que una pareja vive su relación viene determinada tanto por las características particulares de sus miembros como por el contexto cultural y social en el que se inicia y se desarrolla, aspectos que van a ser determinantes en las dinámicas de interacción que se generen en la pareja y, por tanto, en el ajuste y la calidad de la misma.

La influencia de la auto-eficacia social en la calidad de las relaciones románticas ha sido reconocida por su efecto en la percepción de los adolescentes de sí mismos como personas competentes para manejar adecuadamente las exigencias de una relación de pareja (Vargas y Barrera, 2003).

Se reconoce además que la calidad de las relaciones románticas viene influenciada por el apoyo social percibido por parte de la familia, los iguales y la pareja sentimental, como principales procuradores de apego. En algunas investigaciones se demuestra que las personas con apego seguro muestran mayor apoyo mutuo, orientación hacia la pareja y cohesión, mayores niveles de confianza, intimidad, sensibilidad y sentimientos e ideas compartidas (Simpson et al., 1992).

El grupo familiar ejerce funciones de apoyo, potenciando la capacidad para desarrollar relaciones sociales positivas (Alonso y Román, 2005) y facilitando claves para la construcción de representaciones globales acerca del funcionamiento de las interacciones sociales (Rubin, 2004). De hecho, los adolescentes que se sienten apoyados por sus padres tienden a informar de una autoestima social positiva, perciben que tienen facilidad para hacer amigos y disfrutan de sus relaciones sociales, lo cual favorece la aceptación por sus compañeros y compañeras (Martínez y Fuertes, 1999) y el aprendizaje de herramientas útiles para desenvolverse de forma satisfactoria en las relaciones románticas.

No obstante, en la adolescencia, la evidencia empírica indica que disminuye la percepción de los padres como principal fuente de apoyo mientras que aumenta la necesidad de aceptación de las amistades (Collins y Steinberg, 2006), que 
adquieren mayor importancia como fuente de información, compañía, apoyo, retroalimentación y como modelos de comportamiento, capacitándoles a su vez para experimentar diferentes formas de amistad e identidad (Overbeek, Vollebergh, Engels, y Meeus, 2003). El reconocimiento social que proporciona el grupo de iguales tiene claros beneficios para el adolescente, puesto que incide en el sentimiento de integración social, en la autoestima, así como en la evaluación general de satisfacción vital (Ladd yTroop-Gordon, 2003). Además, el papel de la amistad proporciona el contexto social propicio para el establecimiento de las relaciones románticas y ejerce influencia en la elección de la pareja, en las expectativas y en el comportamiento considerado apropiado (Brown, 1999).

Los objetivos de este estudio son describir las características fundamentales de las relaciones románticas analizar la influencia que la auto-eficacia social y el apoyo social tienen sobre la calidad de las relaciones románticas adolescentes, considerándose las posibles diferencias a razón del sexo y el nivel educativos de los participantes.

\section{METODOLOGÍA}

\section{Participantes}

Este estudio toma una muestra de 209 participantes ( $50.7 \%$ chicas), con edades de 12 a 18 años ( $\mathrm{M}=14.28$; D.T=1.21), escolarizados en Educación Secundaria Obligatoria (el $12 \%$ en $1^{\circ}$, el $39.7 \%$ en $2^{\circ}$, el $31.1 \%$ en $3^{\circ}$ y el $17.2 \%$ en $4^{\circ}$ ). El $68.7 \%$ tiene o ha tenido alguna experiencia sentimental y la duración media de dichas relaciones es de 21.39 semanas (rango de duración $=95$ ).

\section{Instrumentos}

Escala socio-demográfica compuesta por cinco preguntas abiertas que recogían información sobre las características socio-demográficas de los encuestados y sus familias: centro educativo, edad, sexo y curso.

Escala de auto-eficacia social en adolescentes (S-EFF) (Connolly, 1989). Está compuesta por 25 ítems medidos en escala Likert de 7 puntos (1= completo desacuerdo; 7 = completo acuerdo) que evalúa la percepción de auto-eficacia en la cohesión social en cinco dimensiones: Asertividad social (p.e. Inicias una conversación con un chico/a desconocido; $\alpha=.74$ ), Comportamiento público (Te pones en situaciones sociales nuevas; $\alpha=.78$ ), Participación grupal (Participas en actividades grupales; $\alpha=.71)$, Amistad e intimidad (Cuentas tus sentimientos a otro chico/a; $\alpha=.72$ ) y Dar y recibir ayuda (Pides a algún/a compañero/a ayuda cuando lo necesitas; $\alpha=.66$ ).

Cuestionario de Apoyo social (Van Aken, 2001), compuesto por 22 ítems medidos en escala Likert de 7 puntos $(1=$ completo desacuerdo; $7=$ completo acuerdo). Este cuestionario evalúa las dimensiones del apoyo social (apoyo emocional, autonomía, información, metas y aceptación) en el adolescente en 
relación con cuatro fuentes de apoyo: familiar (p.e. Algún familiar demuestra que me quiere; $\alpha=.72$ ), adulto significativo (Algún adulto demuestra que me quiere; $\alpha=.88$ ), amigo/a (Mi amigo/a demuestra que me quiere; $\alpha=.78)$ y novio/a (Mi novio/a demuestra que me quiere; $\alpha=.92)$.

EPO-Peer (Fulligni y Eccles, 1993), compuesto por 4 ítems medidos en escala Likert de 7 puntos ( $1=$ completo desacuerdo; $7=$ completo acuerdo $)$ que evalúan la frecuencia con la que realizaban comportamientos transgresivos para mantener la amistad (p.e. ¿Cuántas veces desobedeces a tu familia para mantener a tus amistades?; $\alpha=.67)$.

Dating Questionnaire (Connolly, Pepler, Craig, y Taradash, 2000) adaptado al español, compuesto por 11 ítems medidos en escala Likert de 7 puntos $(1=$ completo desacuerdo; 7 = completo acuerdo) que evalúan dimensiones cuantitativas y cualitativas de las primeras relaciones sentimentales. Cuatro ítems de respuesta múltiple sobre la situación sentimental, número de relaciones de pareja, duración de las mismas, y tiempo que comparten, (p.e. En este momento salgo con alguien) y siete ítems dicotómicos (verdadero/falso) analizan las actividades sociales (p.e. Quedo con mi pareja para estudiar).

Network Relationships Inventory (Furman y Buhrmester, 2009), compuesto por 15 ítems en escala Likert ( $1=$ completo desacuerdo; $7=$ completo acuerdo) que evalúan la percepción de los adolescentes respecto a la calidad de su relación sentimental en cuatro dimensiones: comunicación (p.e. Cuento todo a mi novio/a; $\alpha=.89$ ), expectativas de futuro (Seguro que esta relación continuará a pesar de todo; $\alpha=.83$ ), compañía/intimidad (Pasotiempo libre con mi novio/a; $\alpha=.77$ ) y los conflictos en pareja (Mi novio/a y yo nos ponemos de los nervios; $\alpha=.79)$.

EPO-couple (Fulligni y Eccles,1993), compuesto por diez ítems medidos en escala Likert de 7 puntos $(1=$ completo desacuerdo; $7=$ completo acuerdo) que evalúan la tendencia al comportamiento transgresivo (¿Cuántas veces desobedeces a tu familia por tu novio/a?; $\alpha=.83$ ) y el desequilibrio de poder en la pareja (¿Cuántas veces te has enfadado con tu pareja por su ropa o sus amigos/as?; $\alpha=.88)$.

Escala de Manejo del Afecto y Diferencias (MADS) (Arellano y Markman,1995), compuesta por 32 ítems, medidos en escala Likert de 7 puntos (1= completo desacuerdo; 7 = completo acuerdo) que evalúan la utilización de estrategias para negociar los conflictos de pareja. Se han utilizado cinco sub-escalas: nivelación (p.e. Ayudo a mi pareja a entenderme; $\alpha=.88$ ), detener acciones (Cuando los conflictos se van de las manos, prefiero hablar en otro momento; $\alpha$ $=.68$ ), centrar enfoque (Cuando nos desviamos del tema, trato de que volvamos al mismo; $\alpha=.83$ ), retirada (Cuando surgen problemas, mi pareja se distancia; $\alpha$ $=.77$ ) y progresividad negativa (Soy incapaz de salir de discusiones acaloradas; $\alpha=.70)$.

Escala de Celos románticos (White,1976), compuesta por seis ítems medidos en escala Likert de 7 puntos $(1=$ completo desacuerdo; $7=$ completo acuerdo $)$ que evalúan la existencia de celos en las relaciones de pareja (p.e. ¿Cuánto de 
celoso/a eres?; $\alpha=.84)$.

Control in Dating Relationships (Stets, 1993), compuesta por nueve ítems medidos en escala Likert ( $1=$ completo desacuerdo; $7=$ completo acuerdo) que evalúan indicadores de control en la pareja (p.e. Hago que él/ella haga lo que yo quiero; $\alpha=.88)$.

\section{Procedimiento}

Los cuestionarios auto-informe fueron cumplimentados en horario escolar previa autorización de los centros colaboradores. La persona encargada de suministrarlos informó de su carácter voluntario e individual, así como del tratamiento anónimo y confidencial de los datos. La cumplimentación osciló entre los 30 y 40 minutos.

\section{RESULTADOS}

\section{Características de las relaciones románticas adolescentes}

Los resultados mostraron que un $68.5 \%$ de los encuestados tenía o había tenido alguna experiencia sentimental, sin diferencias significativas entre sexos. No obstante, el porcentaje de implicados en este tipo de relaciones aumentaba a medida que avanzaba la edad, de forma que mientras que en $1^{\circ}$ ESO un $6.3 \%$ afirmaba no tener experiencia sentimental previa, en $4^{\circ}$ ESO disminuía al 2.0\%, $\left(\chi^{2}(6,205)=19.103 ; p<.01\right)$. No obstante, los valores de los índices V de Cramer .216 y de Lambda .077 indicaban que esta relación podía estar sesgada por el tamaño muestral.

Los chicos y chicas habían tenido entre una y 12 parejas, con una media de 3.23 (D.T. $=2.60)$, siendo las relaciones actuales más duraderas (21.34 semanas) que las pasadas (14.90 semanas), sin hallar diferencias significativas entre niveles educativos, ni a razón de sexo.

La mayoría de los participantes pasaban de 4-8 horas semanales con sus parejas. No se observaron diferencias significativas entre la variable tiempo y el sexo y el curso. Un $80.3 \%$ afirmaba que salía con alguien en pandilla con momentos a solas, y entre las actividades compartidas destacaban el pasar tiempo libre en pareja asistiendo a eventos (67.2\%) y quedar en casa para salir juntos $(62.1 \%)$, sin diferencias significativas entre sexos. La actividad "quedo en mi casa o la suya para salir juntos" se veía influida por el nivel educativo $\left(\chi^{2}(2,134)=11.735 ; p<\right.$ .01). La puntuación de la prueba $\mathrm{V}$ de Cramer .008 y de Lambda .416 indicaban que la relación entre las variables no estaba sesgada por el tamaño muestral.

La Figura1 muestra las medias en los factores de calidad de las relaciones de parejas. Las puntuaciones medias en las escalas positivas estaban en torno a 5 y las negativas en torno a 3, lo que indicaba que los adolescentes tenían una percepción de calidad de sus parejas media-alta, sin que hubiera diferencias significativas en función del sexo y el curso. 


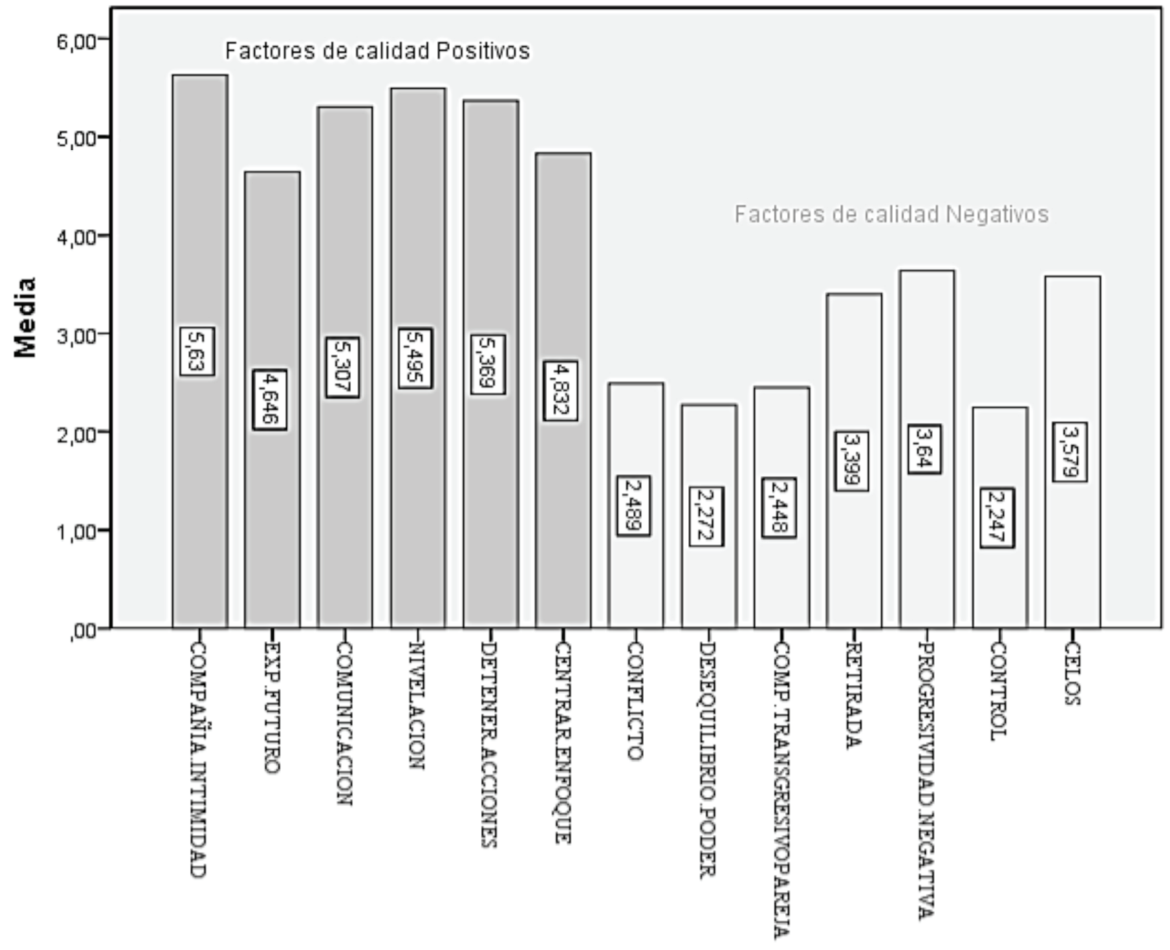

Figura 1. Factores de calidad de las relaciones románticas.

\section{Auto-eficacia social y Apoyo social percibido}

La Tabla 1 presenta las puntuaciones obtenidas en las escalas de auto-eficacia social y el comportamiento transgresivo entre iguales. Los y las adolescentes reportan índices altos de auto-eficacia social rondando una media de 5, sin embargo la media de comportamiento transgresivo entre iguales gira en torno a 3. Las chicas tenían mayores medias en las dimensiones de auto-eficacia social y menor en el comportamiento transgresivo (2.66). Los resultados mostraron diferencias significativa en función del sexo en la participación social $(t=3.816 ; p<.01)$, siendo las chicas las que tenían medias más altas (6.10), y en la asertividad social $(t=4.148 ; p<.01)$ en la cual los chicos tenían una media de 4.60 y las chicas de 5.25. Respecto al nivel educativo, el análisis ANOVA de la variable auto-eficacia social indicaba que solo existían diferencias significativas en Comportamiento público $(F=4.334 ; p<.01)$ y en Dar y recibir ayuda $(F=2.705 ; p=.047)$ entre $2^{\circ} \mathrm{ESO}$ y $3^{\circ} \mathrm{ESO}$.

El siguiente análisis estaba dirigido al apoyo social percibido por las principales figuras de apego, relacionándolo con el sexo y el nivel educativo. En la Figura 2 se observa que los jóvenes percibían gran apoyo por parte de todos los 
procuradores de apego, destacando el apoyo familiar, seguido del de los amigos/ as. La prueba $t$ de Student apuntaba que el Apoyo familiar $(t=-2.535 ; p=.012)$ variaba según el sexo, siendo los chicos los que mostraban una media mayor (chicos 102.97 vs. chicas 97.34). En las demás escalas no se observaron diferencias significativas.

Tabla 1. Auto-Eficaia Social y Comportamiento Transgresivo entre Iguales por Sexo

\begin{tabular}{ccccc}
\cline { 3 - 5 } & & $N$ & Media & DT \\
\hline \multirow{2}{*}{ Asertividad } & Chica & 97 & 5.25 & 1.07 \\
\cline { 2 - 5 } & Chico & 105 & 4.60 & 1.12 \\
\hline \multirow{2}{*}{ Comportamiento público } & Chica & 98 & 5.51 & .92 \\
\cline { 2 - 5 } & Chico & 95 & 5.08 & 1.11 \\
\hline \multirow{2}{*}{ Participación } & Chica & 96 & 6.09 & .72 \\
\cline { 2 - 5 } & Chico & 98 & 5.62 & .97 \\
\hline \multirow{2}{*}{ Amistad e intimidad } & Chica & 100 & 5.22 & .76 \\
\cline { 2 - 5 } & Chico & 101 & 5.10 & 1.03 \\
\hline \multirow{2}{*}{ Dar y recibir ayuda } & Chica & 100 & 5.22 & .91 \\
\cline { 2 - 5 } & Chico & 103 & 5.10 & .98 \\
\hline \multirow{2}{*}{ Comportamiento Transgresivo } & Chica & 100 & 2.66 & 1.28 \\
\cline { 2 - 5 } & Chico & 104 & 3.22 & 1.34 \\
\hline
\end{tabular}

Los adolescentes que percibían un mayor apoyo eran los escolarizados en $1^{\circ} \mathrm{ESO}$, seguido de los de $4^{\circ} \mathrm{ESO}$, siendo los de $3^{\circ} \mathrm{ESO}$ los que menos apoyo percibían. Se observa un auge significativo de la percepción de apoyo del novio/a en $4^{\circ} \mathrm{ESO}$ (ver Figura 2).

$\mathrm{Al}$ analizar el ANOVA de los tipos de apoyo relacionándolo con el nivel educativo encontramos que todos los tipos de apoyo advertían diferencias significativas, Apoyo familiar $(F=3.955 ; p<.01)$, Apoyo de otro adulto $(F=3.532 ; p=$ $.016)$, Apoyo amigo/a $(F=6.919 ; p<.01)$ y Apoyo novio/a $(F=4.504 ; p<.01)$. La prueba Post hoc indica que en el apoyo familiar $1^{\circ} \mathrm{ESO}$ difería con $2^{\circ} \mathrm{ESO}$, $3^{\circ} \mathrm{ESO}$ y $4^{\circ} \mathrm{ESO}$. En apoyo de otro adulto $1^{\circ} \mathrm{ESO}$ difería con $2^{\circ} \mathrm{ESO}$ y $4^{\circ} \mathrm{ESO}$. En Apoyo del amigo/a, $1^{\circ} \mathrm{ESO}$ difería con $2^{\circ} \mathrm{ESO}, 3^{\circ} \mathrm{ESO}$ y $4^{\circ} \mathrm{ESO}$. Por último, en Apoyo del novio/a $3^{\circ} \mathrm{ESO}$ difería con $1^{\circ} \mathrm{ESO}, 2^{\circ} \mathrm{ESO}$ y $4^{\circ} \mathrm{ESO}$. 


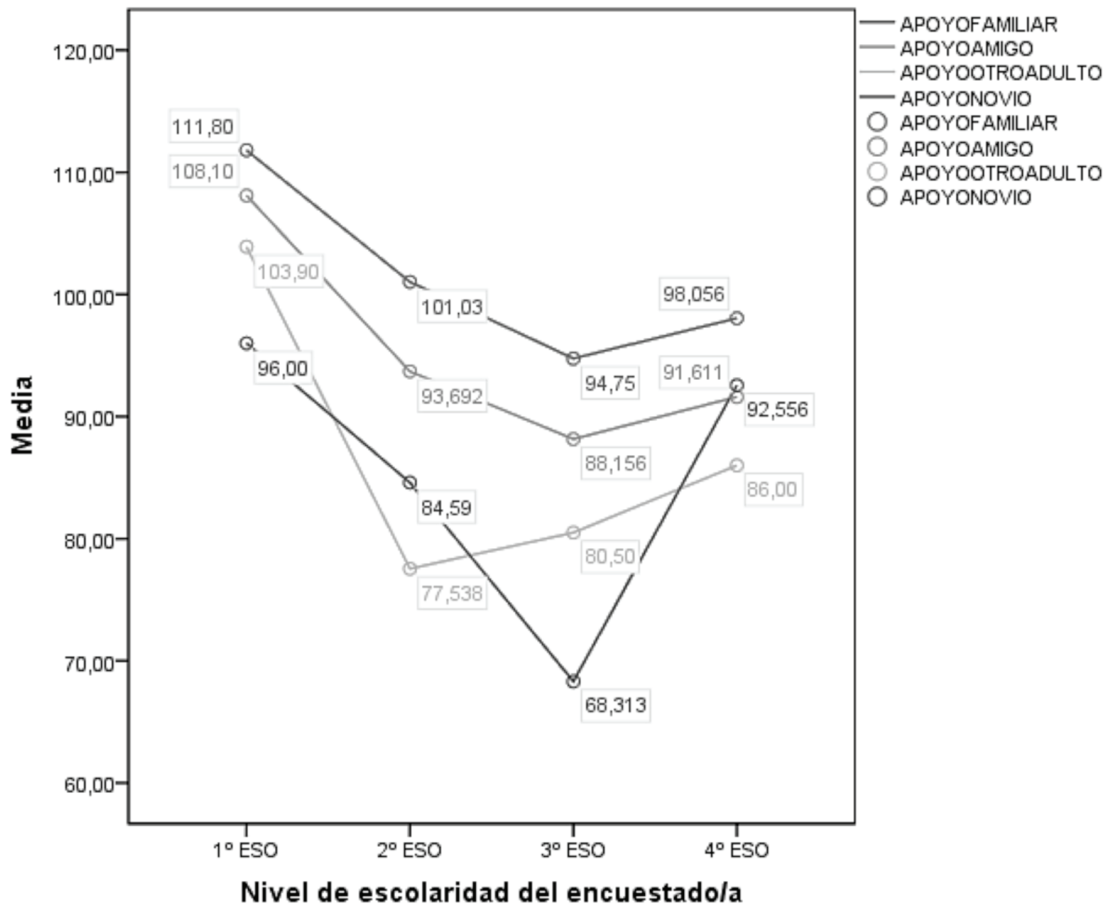

Figura 2. Apoyo social por nivel educativo.

Influencia de las relaciones sociales en la calidad de las relaciones románticas

Para conocer la relación entre las relaciones sociales y la calidad de las parejas románticas adolescentes se realizaron dos correlaciones de Pearson; la primera entre la relación con los iguales, la familia y la propia pareja con los indicadores positivos de calidad de la pareja, y la segunda entre la relación con los iguales, la familia y la pareja con los indicadores negativos de calidad de las parejas.

En la Tabla 2 se muestran los resultados de la primera correlación de Pearson realizada, entre los cuales se han observado relaciones positivas y significativas entre la auto-eficacia y la compañía e intimidad, la nivelación y la centración de enfoque. Se han obtenido relaciones negativas y significativas entre el comportamiento transgresivo entre iguales y los indicadores de calidad de pareja como la nivelación y detener acciones. Se observaron relaciones positivas y significativas entre el apoyo familiar y el apoyo del novio/a, entre el apoyo del mejor amigo/a y el apoyo del novio/a, y entre el apoyo del novio/a y la calidad de las relaciones de pareja en indicadores como la compañía e intimidad, la comunicación, las expectativas de futuro en pareja, el detener acciones y la centración. 
Tabla 2. Correlación entre la Relación con los Iguales y Familia y los Factores Positivos de Calidad de las Relaciones Románticas

\begin{tabular}{|c|c|c|c|c|c|}
\hline & $\begin{array}{l}\text { Auto } \\
\text { eficacia }\end{array}$ & $\begin{array}{c}\text { Comp. } \\
\text { Transgresivo } \\
\text { iguales }\end{array}$ & $\begin{array}{l}\text { Apoyo } \\
\text { Familiar }\end{array}$ & $\begin{array}{c}\text { Apoyo } \\
\text { Amigo/a }\end{array}$ & $\begin{array}{c}\text { Apoyo } \\
\text { Novio/a }\end{array}$ \\
\hline \multirow{2}{*}{$\begin{array}{c}\text { Compañía e } \\
\text { Intimidad }\end{array}$} & $.286^{(* *)}$ & .014 & .137 & .228 & $.343^{(* *)}$ \\
\hline & $N=100$ & $N=118$ & $N=101$ & $N=101$ & $N=79$ \\
\hline \multirow{2}{*}{ Comunicación } & .187 & -.063 & .114 & .148 & $.341^{(* *)}$ \\
\hline & $N=110$ & $N=130$ & $N=110$ & $N=109$ & $N=90$ \\
\hline \multirow{2}{*}{ Exp.Futuro } & .092 & .060 & .148 & .278 & $.402^{(* *)}$ \\
\hline & $N=109$ & $N=129$ & $N=110$ & $N=111$ & $N=90$ \\
\hline \multirow{2}{*}{ Nivelación } & $.209^{(*)}$ & $-.336^{(* *)}$ & .059 & .131 & .072 \\
\hline & $N=94$ & $N=106$ & $N=91$ & $N=90$ & $N=73$ \\
\hline \multirow{2}{*}{$\begin{array}{l}\text { Detener } \\
\text { acciones }\end{array}$} & .123 & $-.192^{(*)}$ & .131 & .115 & $.229^{(*)}$ \\
\hline & $N=110$ & $N=132$ & $N=112$ & $N=111$ & $N=89$ \\
\hline \multirow{2}{*}{$\begin{array}{l}\text { Centración del } \\
\text { Enfoque }\end{array}$} & $.266^{(* *)}$ & .016 & .090 & .94 & $.021^{(* *)}$ \\
\hline & $N=106$ & $N=125$ & $N=106$ & $N=105$ & $N=85$ \\
\hline \multirow{2}{*}{$\begin{array}{c}\text { Apoyo del } \\
\text { Novio/a }\end{array}$} & .123 & .140 & $.415^{(* *)}$ & $.568^{(* *)}$ & 1 \\
\hline & $N=95$ & $N=112$ & $N=111$ & $N=110$ & $N=113$ \\
\hline
\end{tabular}

En la Tabla 3 se presentan los resultados de la correlación de Pearson realizada entre la relación con los iguales, la familia y con la propia pareja, y los factores negativos de calidad de las relaciones románticas. Se observaron relaciones positivas y significativas entre el comportamiento transgresivo entre los iguales y los indicadores negativos de calidad de pareja como el conflicto, el comportamiento transgresivo en pareja, el desequilibrio de poder, los celos y el control. En lo referente al apoyo social, se observan relaciones positivas y significativas entre el apoyo familiar y la progresividad negativa y la retirada en momentos acalorados en pareja; entre el apoyo del amigo/a y el comportamiento transgresivo, los celos y el control; y entre el apoyo del novio/a y el control en la pareja. 
Tabla 3. Correlación entre la Relación con los Iguales y Familia y los Factores Negativos de Calidad de las Relaciones Románticas

\begin{tabular}{|c|c|c|c|c|c|}
\hline & $\begin{array}{l}\text { Auto } \\
\text { eficacia }\end{array}$ & $\begin{array}{c}\text { Comp. } \\
\text { Transgresivo } \\
\text { iguales }\end{array}$ & $\begin{array}{l}\text { Apoyo } \\
\text { Familiar }\end{array}$ & $\begin{array}{l}\text { Apoyo de } \\
\text { Amigo/a }\end{array}$ & $\begin{array}{c}\text { Apoyo } \\
\text { Novio/a }\end{array}$ \\
\hline \multirow{2}{*}{ Conflicto } & -.042 & $.304^{(* *)}$ & -.029 & .119 & .071 \\
\hline & $N=96$ & $N=112$ & $N=98$ & $N=97$ & $N=76$ \\
\hline \multirow{2}{*}{$\begin{array}{c}\text { Comp. } \\
\text { Transgresivo en } \\
\text { pareja }\end{array}$} & -.007 & $.525^{(* *)}$ & 053 & $.234^{(*)}$ & .154 \\
\hline & $N=109$ & $N=132$ & $N=112$ & $N=112$ & $N=92$ \\
\hline \multirow{2}{*}{$\begin{array}{c}\text { Desequilibrio de } \\
\text { Poder }\end{array}$} & -.025 & $265^{(* *)}$ & 018 & .169 & .052 \\
\hline & $N=108$ & $N=128$ & $N=110$ & $N=110$ & $N=90$ \\
\hline \multirow{2}{*}{ Celos } & .028 & $287^{(* *)}$ & 180 & $.226^{(*)}$ & .142 \\
\hline & $N=107$ & $N=127$ & $N=112$ & $N=111$ & $N=89$ \\
\hline \multirow{2}{*}{ Control } & -.089 & $334^{(* *)}$ & .144 & $.260^{(* *)}$ & $.252^{(*)}$ \\
\hline & $N=107$ & $N=128$ & $N=111$ & $N=111$ & $N=87$ \\
\hline \multirow{2}{*}{ Retirada } & -.036 & .090 & $200^{(*)}$ & .167 & .106 \\
\hline & $N=103$ & $N=122$ & $N=106$ & $N=105$ & $N=84$ \\
\hline \multirow{2}{*}{$\begin{array}{c}\text { Progresividad } \\
\text { Negativa }\end{array}$} & .020 & .157 & $.235^{(*)}$ & $.256^{(*)}$ & .124 \\
\hline & $N=95$ & $N=114$ & $N=100$ & $N=100$ & $N=76$ \\
\hline
\end{tabular}

\section{DISCUSIÓN Y CONCLUSIONES}

Este estudio pretendía analizar las variables cuantitativas relacionadas con las relaciones románticas en adolescentes. Los resultados han mostrado que casi un $70 \%$ de los participantes tenía o había tenido alguna experiencia sentimental, confirmando la importancia de las primeras relaciones románticas en la adolescencia. (Steinberg, 2008). La literatura señala que dicha importancia aumenta con la edad (Tani y Fonzi, 2005), y aunque en nuestro estudio no aparecen diferencias significativas, sí se muestra una tendencia a aumentar el número de chicos y chicas que tenían pareja.

En cuanto al análisis de las características de las relaciones románticas adolescentes, los resultados mostraron que los encuestados habían mantenido unas tres parejas a lo largo de su vida. Este dato parecía ser independiente del sexo de los implicados, al igual que en otras investigaciones desarrolladas al respecto en el ámbito internacional (Menesini y Nocentini, 2008). También se han obtenido datos en relación a la solidez de las relaciones de pareja adolescente, cuyos resultados nos acercan a la perspectiva de algunos investigadores (Collins, 2003) que consideraban que las relaciones de pareja adolescente eran triviales, transitorias y carentes de compromiso. No obstante, hemos encontrando que la duración de las relaciones que mantenían en la actualidad era mayor que la de las relaciones pasadas, y esto podría indicar que muchas de estas relaciones iban haciéndose 
más estables a medida que se tenían más experiencias de pareja (Carver, Joyner, y Udry, 2003).

En relación al tiempo y las actividades compartidas en pareja, los datos señalaron que la mayoría de las parejas comparten de cuatro a ocho horas semanales y tres de cada cuatro sale con su pareja en pandilla con momentos a solas, por lo que comprobamos que el papel de los amigos y amigas es fundamental en las relaciones románticas adolescentes, puesto que le proporciona el contexto en el que se establecen dichas relaciones (Brown, 1999).

En la calidad de las relaciones sentimentales, los resultados apuntan que los adolescentes perciben tener una alta satisfacción con sus relaciones de pareja, sintiéndose acompañados, con buena comunicación y con unas expectativas positivas sobre la continuidad del vínculo amoroso. No obstante, los chicos muestran mayor tendencia a la transgresión, a los celos y al control de la pareja.

Los factores de calidad positivos tienden a ascender con la edad, excepto la nivelación y el enfoque de centración, que son estrategias fundamentales a la hora de resolver conflictos. De acuerdo a estudios previos, estos datos junto al descenso que sufren los factores negativos en $4^{\circ} \mathrm{ESO}$, nos lleva a pensar que conforme vaya transcurriendo la adolescencia, las relaciones de pareja se van haciendo más estables, transmitiendo mayor seguridad y apoyo, pero también aumentan los conflictos de pareja (Tani y Fonzi, 2005).

Este estudio también pretendía conocer la auto-eficacia social y el apoyo social que los adolescentes percibían para evaluar las relaciones de los iguales y la familia. Los participantes tuvieron puntuaciones altas en todas las dimensiones de auto-eficacia y bajas en comportamiento transgresivo, siendo por tanto que la relación con los iguales es satisfactoria (Gifford-Smith y Brownell, 2003).

En lo referente al apoyo social, los participantes perciben un gran apoyo por parte de todas las figuras de apego. Los chicos y chicas perciben mayor apoyo de la familia seguido de los amigos y amigas en todos los niveles educativos. Los resultados obtenidos son contrarios a algunos estudios (Overbeek, Volleberg, Engels y Meeus, 2003, que asumían que declinaba la percepción de los padres como principal fuente de apoyo mientras aumentaba la de los iguales. No obstante otros estudios (Gray y Steinberg,1999) coinciden en la importancia de la familia para el ajuste social y emocional de los jóvenes. En el último nivel educativo estudiado se produce un auge del apoyo del novio/a que se puede traducir en que las relaciones de pareja van ascendiendo en la jerarquía de apego a lo largo de la adolescencia (Martínez y Fuertes, 1999).

Al analizar la relación e influencia de la auto-eficacia social y el apoyo social en la calidad de las relaciones románticas adolescentes, los resultaron indicaban que los y las adolescentes que eran más auto-eficaces socialmente, contaban con más habilidades para manejar adecuadamente las exigencias de una relación de pareja y se sentían más satisfechos (Vargas y Barrera, 2003).

Se ha observado que a medida que crecía el apoyo familiar y de los amigos/ as, también lo hacía considerablemente la percepción de apoyo por parte de la pareja, y es que el grupo familiar ejerce funciones de apoyo que potencian la 
capacidad para desarrollar relaciones sociales positivas (Alonso y Román, 2005). Además encontramos que cuando aumentan los comportamientos transgresivos entre los iguales también lo hacen de forma considerable los indicadores negativos de calidad en pareja, al mismo tiempo que reduce la nivelación y el detener acciones en momentos acalorados. Estos datos pueden ser explicados por el hecho de que los jóvenes que no tienen una percepción de autoeficacia social positiva son más vulnerables al estrés (Pastorelli, Caprara, Barbaranelli, Rola, Rozsa, y Bandura, 2001).

Por último, concluimos que los adolescentes que percibían mayor apoyo por parte de la pareja, se mostraban más hábiles en las estrategias sociales. Aunque con frecuencia, también hacía que aumentara el control ejercido sobre esta.

Para finalizar, desearíamos dejar constancia de la potencialidad y limitaciones de este estudio. En lo referente a la potencialidad, este estudio contribuye al fortalecimiento de los conocimientos existentes sobre el rol de la auto-eficacia social, así como del efecto que tiene el apoyo social para mantener relaciones románticas de calidad. Además los hallazgos encontrados pueden contribuir a encaminar el trabajo preventivo en la calidad de las relaciones románticas adolescentes.

Las principales limitaciones encontradas han sido el pequeño tamaño muestral que no nos permite hacer generalizaciones, ni transferir resultados a otras poblaciones.

\section{AGRADECIMIENTOS}

Este estudio se ha realizado dentro del proyecto Estudio de la competencia para la gestión de la vida social y su estabilidad en estudiantes de primaria y secundaria de Andalucía" financiado por la Fundación Pública Andaluza Centro de Estudios Andaluces (PRY040/14) (Consejería de la Presidencia, Junta de Andalucía).

\section{REFERENCIAS}

Alonso, J., y Román, J. (2005). Prácticas educativas familiares y autoestima. Psicothema, 17, 76-82.

Arellano, C. M., y Markman, H. J.(1995). The managing affect and differences scale (MADS): A self-report measure assessing conflict management in couples. Journal of Family Psychology, 9, 319-334.

Brown, B. B. (1999). "You're going out with who?" Peer group influences on adolescent romantic relationships. En W. Furman, B. B. Brown y C. Feiring (Eds.), The development of romantic relationships in adolescence (pp. 291329). New York: Cambridge.

Carver, K., Joyner, K., y Udry, J. R. (2003). National Estimates of Adolescent Romantic Relationships. En P. Florsheim (Ed.), Adolescent Romantic Relations and Sexual Behavior. Theory, Research, and Practical Implications (pp. 23-56). Mahwah, New Jersey: Lawrence Erlbaum Associates. 
Collins, A. W. (2003).More than myth:the developmental significance of romantic relationships during adolescence. Journal of research on adolescence, 13, 1-24.

Collins, W. A., y Steinberg, L. (2006). Adolescent development in interpersonal context. En N. Eisenberg (Ed.), Social, emotional, and personality development. Handbook of child psychology (pp.1003-1067). New York: Woley.

Connolly, J. (1989). Social Self-Efficacy in adolescence: Relations with selfconcept, social adjustment and mental health. Journal of Behavioral Science, 21, 258-269.

Connolly J., Pepler D., Craig W., y Taradash A. (2000). Dating experiences of bullies in early adolescence. Child Maltreatment, 5, 299-310.

Engels, R., Finkenauer, C., Meeus, W., y Dekovic, M. (2001). Parental attachment and adolescents emotional adjustment: The associations with social skills and relational competence. Journal of Couseling Psychology, 48, 428439.

Feiring, C., Simon,V., y Cleland, C. (2009). Childhood Sexual Abuse, Stigmatization, Internalizing Symptoms, and the Development of Sexual Difficulties and Dating Aggression. Journal of Consulting and Clinical Psychology, 77, 127-137.

Fuligni, A. J., y Eccles, J. S.(1993). Perceived Parent-Child Relationships and Early Adolescents' Orientation Toward Peers. Developmental Psychology, 29, 622-632.

Furman,W., y Buhrmester, D. (2009). The Network of Relationships Inventory: Behavioral Systems Version. Journal of Behavior Development, 33, 470-478

Gorrese, A., y Ruggieri, R. (2012). Peer attachment: a meta-analytic review of gender and age differences and associations with parent attachment. Journal of Youth Adolescence, 41, 650-672.

Gray, M., y Steinberg, L. (1999). Adolescent romance and the parent-child relationship: A contextual perspective. En W. Furman, B. B. Brown, y C. Feiring (Eds.), The development of romantic relationships in adolescence (pp. 235262). New York: Cambridge.

Lewis, S. F., y Fremouw, W. (2000). Dating violence: a critical review of the literature. Clinical Psychology Review, 21, 105-127.

Martínez, J. L., y Fuertes, A. (1999). Factores personales, familiares y relacionales implicados en la estabilidad de relaciones de pareja adolescentes. Infancia y Aprendizaje, 88, 85-105.

Menesini, E., y Nocentini, A. (2008). Comportamenti aggressivinel le prime esperienze sentimentali in adolescenza. Giornale Italiano di Psicologia, 2, 407-434.

Moreno, D., Estévez, E., Murgui, S., y Musitu, G. (2009). Reputación social y violencia relacional en adolescentes: el rol de la soledad, la autoestima y la satisfacción vital. Psicothema, 4, 537-542

Overbeek, G., Vollebergh, W., Engels, C., y Meeus, W. (2003). Parental attachment and romantic relationships. Associations with emotional disturbance 
during late adolescence. Journal of Counseling Psychology, 50, 28-39.

Parke, R. D. (2004). Development in family. Annual Review of Psychology,55, 365-399.

Pastorelli, C., Caprara, G.V., Barbaranelli, C., Rola, J., Rozsa, S., y Bandura, A. (2001). The structure of children's perceived self-efficacy: a cross-national study. Journal of Psychological Assessment, 17, 87-97.

Rubin, K. H., et al. (2004). Attachment, friendship, and psychosocial functioning in early adolescence. Journal of Early Adolescence, 24, 326-356.

Sánchez-Queijada, I., y Oliva, A. (2003). Vínculos de apego con los padres y relaciones con los iguales durante la adolescencia. Revista de Psicología Social, 18, 71-86.

Shorey, R., Febres, J., Brasfield, H., y Stuart, G.(2012). Male dating victimization and adjustment. The moderating role of coping. Journal of Men's Health, 3, 218-228.

Simons, R. L., Chao, W., Conger, R. D., y Elder, G. H. (2001). Quality of parenting as mediator of the effect of childhood defiance on adolescent friendship choices and delinquency: A growth curve analysis. Journal of Marriage and Family, 63, 63-79.

Simpson, J. A., Rholes, W. S., y Nelligan, J. S.(1992). Support seeking and support giving within couples in an anxiety provoking situation: The role of attachment styles. Journal of Personality and Social Psychology, 62, 434446.

Stemberg, L. (2008). Adolescence. New York: McGraw Hill.

Steinberg, L., y Morris, A. S.(2001). Adolescent development. Annual Review of Psychology, 52, 83-110.

Stets, J. E. (1993). Control in dating relationships. Journal of Marriage and the Family, 55, 673-685.

Tani, F., y Fonzi, A. (2005). Relazioni di amicizia e relazioni sentimental in el passaggiodall'adolescenza all'età adulta. Età Evolutiva, 80,90-98.

Van Aken, M. (2001). Cuestionario de Relaciones Interpersonales durante la adolescencia. Universidad de Nijmegen. Holanda.

Vargas Trujillo, E., y Barrera, F. (2003). Actividad sexual y relaciones románticas durante la adolescencia: algunos factores explicativos. Bogotá: Universidad de los Andes, Facultad de Ciencias Sociales.

White, G.L.(1976). The social psychology of romantic jealousy. Tesis doctoral o publicada. Universidad de California, Los Ángeles. 